\title{
Mobility Prediction of Diurnal Users for Enabling Context Aware Resource Allocation
}

\author{
Nandish P. Kuruvatti, Wenxiao Zhou*, Hans D. Schotten \\ Institute for Wireless Communications and Navigation \\ University of Kaiserslautern \\ Email: \{kuruvatti,schotten\}@eit.uni-kl.de, zhouw@rhrk.uni-kl.de*
}

\begin{abstract}
Mobile communication is one of the most ubiquitously used technologies in today's world, evolving towards its fifth generation (5G). Amidst increasing number of devices and traffic volume, one of the key focuses of $5 \mathrm{G}$ is to provide uniform service quality despite high mobility. In real world scenarios, user mobility is not random but rather direction oriented, based on its origin and destination. Further, several users exhibit repeated mobility patterns on daily basis (e.g., office goers, commuters in public transport etc.). Such mobility is termed as Diurnal mobility. Information of such diurnal mobility can assist in improving prediction accuracy of future user location (e.g., cells, routes). Knowledge of future user location will enable the designing of efficient resource management algorithms, aiming to make great service quality follow the user. In the presented work, information of diurnal mobility is used to enhance the accuracy of mobility prediction (next cell prediction as well as route prediction) in a realistic urban scenario. Further, using this context information about future routes and possible coverage holes in them, efficient resource allocation is done to sustain streaming/full buffer services, even in coverage holes. The simulation results show substantial improvements in user throughput as a result of context aware resource allocation, enabled by diurnal user mobility prediction.
\end{abstract}

Index Terms- Mobility prediction, Diurnal mobility, Next cell prediction, Route prediction, Context aware resource allocation

\section{INTRODUCTION}

In the contemporary world, mobile communication has become a widely used technology with its applications expanding to new horizons. Over the years, mobile communication has evolved through several generations of technology and is currently foreseeing inception of its fifth generation $(5 \mathrm{G})$. The ubiquitous usage of mobile communication is reflected by the increasing number of new mobile subscriptions each year [1]. By the year 2020, the number of connected devices are anticipated to be more than 50 billion [1]. Further, the mobile multimedia services are gaining wide popularity, leading to an increased data traffic demand by mobile users [2]. Taking these facts into account, it is forecast that by 2020, mobile traffic volume will be at least a 1000 times larger than the present [1] and the number of connected devices will be 10 to 100 times more[2]. Thus, the key challenges in 5G mobile communication are to support high traffic volume and large number of connected devices. Another key focus of $5 \mathrm{G}$ is to make sure that best service experience follows the user[2], irrespective of high mobility.

Mobility prediction plays an important role in designing of context aware radio resource management (RRM), which aims at providing uniform service quality. Knowledge of future user location (position, route or next cell) can be used to anticipate future data traffic conditions, future events (crowd formation, traffic jams etc.) [3] and appropriately reserve or manage resources to provide optimum service. There are several works in literature focusing on mobility prediction such as, prediction based on distributed markov chains, hidden markov model based prediction [4][5], making use of neural networks and machine learning [6] etc. Majority of these work, consider regular hexagonal cells and intend to predict the next cell for user transition based on different strategies. Further, they consider either straight line mobility of users or random way point mobility, to evaluate the performance of prediction schemes.

In real world scenarios, user mobility is not random but is rather direction oriented. The user direction relies on its origin and destination. Further, there are several users who exhibit similar mobility patterns on daily basis (e.g., office goers, public transport). They tend to regularly traverse a limited set of trajectories, comprising of specific landmarks. Such mobility can be referred to as Diurnal mobility, which constitutes a major portion of mobile users. In this work, users following diurnal mobility are considered and information arising from such mobility (E.g., origin, landmarks, destination) are used to enhance the accuracy of mobility prediction. Further, a realistic urban scenario with irregular base station placements (non hexagonal layout), several roads, crossroads, parks and buildings is considered. The considered layout is also referred to as Madrid grid [7].

In day to day life, there are several instances when a user will run into a coverage hole (e.g., tunnel), where his throughput will be nil. By anticipating the encounter of such a coverage hole in near future, it is possible to allocate more resources suitably and buffer the data before running into coverage hole. In coverage hole, buffered data can be used to sustain streaming/full buffer service. This will enable an uniform service experience for the user, even in deep shadow regions or coverage holes. This work exploits the context information gained from route prediction to anticipate running into a coverage hole and initiates context aware resource allocation. Thus, substantial improvements are observed in throughput of the user, sustaining streaming/full buffer service 
even in case of coverage holes. The remainder of this paper is organized as follows: Section II deals with system model. Section III discusses next cell prediction and route prediction, Section IV deals with context exploitation, Section V discusses simulation results and Section VI provides a conclusion and indicates future work.

\section{System Model}

The mobility of users in day to day scenarios is not random. Majority of the user movements are rather direction oriented, depending on origin and destination of user. As an example, consider figure 1, a user traverses through following 5 landmarks on daily basis. The user starts from his home in morning, travels to his office, then to restaurant at afternoon, then to library and gym in evening, before returning home. There might be more than one path that exist between two landmarks and sequence of landmarks traversed by user might be different as well. But his trajectories are confined to specific set of landmarks. Such mobility can be termed as diurnal mobility. This type of mobility can be observed by monitoring the users for a specific period of time (e.g., several business days), before classifying them as diurnal mobile users. The information about the origin and destination of such users will assist in improving the accuracy of mobility prediction.

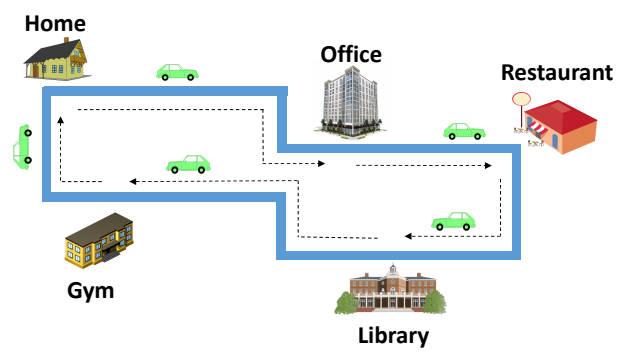

Fig. 1. Diurnal mobility

The system model used here is referred to as Madrid grid [7] and is depicted in figure 2. The system model captures the realistic urban scenario comprising of several buildings, roads, cross roads, parks and traffic lights. This 3D system model is created using game engine called UNITY 3D. There are 12 micro base stations positioned at various sites in the considered model as opposed to simple conventional hexagonal cell layout. There are 25 crossroads present in the considered layout. There are 7 landmarks at different crossroads namely: home, university, gym, office, and corner points (North East, South East and South West) as shown in figure 2. The mobile user is assumed to follow diurnal mobility and can take 10 different paths with different probabilities as given in table I. Each path is defined by sequence of crossroads, including the landmarks.

\section{DiURnal Mobility PREdiction}

\section{A. Next Cell Prediction}

The mobile user connects to different base stations as it traverses a path. With each path, a unique sequence of base

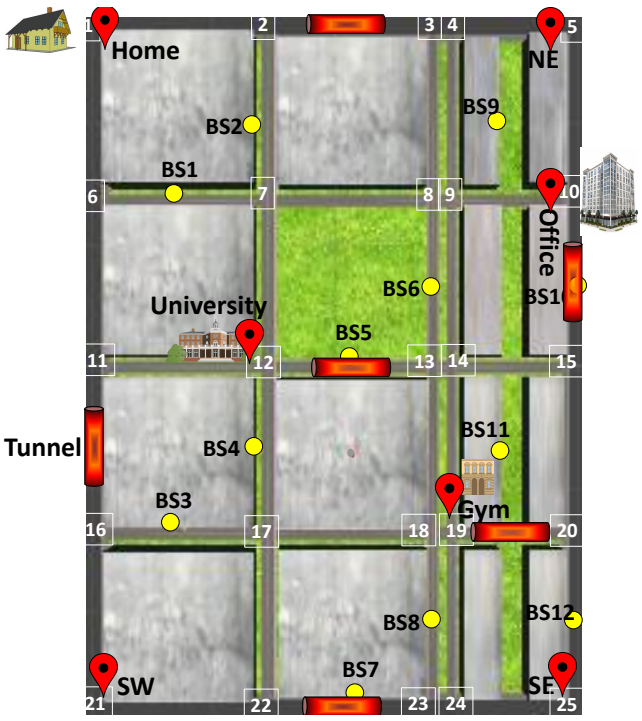

Fig. 2. System model

TABLE I

PATHS TAKEN BY THE MOBILE USER

\begin{tabular}{|c|c|c|}
\hline Nr. & $\begin{array}{l}\text { Sequence of crossroads } \\
\end{array}$ & \%age \\
\hline 1 & 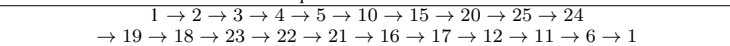 & $10 \%$ \\
\hline 2 & 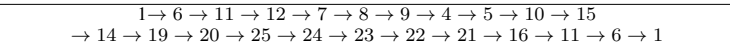 & $8 \%$ \\
\hline 3 & 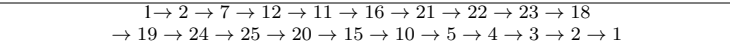 & $7 \%$ \\
\hline 4 & $\begin{aligned} 1 \rightarrow 6 & \rightarrow 7 \rightarrow 12 \rightarrow 13 \rightarrow 14 \rightarrow 19 \rightarrow 18 \rightarrow 17 \rightarrow 16 \\
\rightarrow 21 \rightarrow 22 \rightarrow 23 & \rightarrow 18 \rightarrow 19 \rightarrow 24 \rightarrow 25 \rightarrow 20 \rightarrow 15 \rightarrow 10 \rightarrow 5 \rightarrow 4 \rightarrow 3 \rightarrow 2 \rightarrow 1\end{aligned}$ & $5 \%$ \\
\hline 5 & 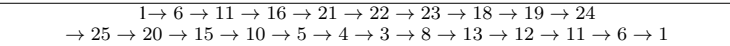 & $5 \%$ \\
\hline 6 & $\begin{aligned} & \rightarrow 2 \rightarrow 3 \rightarrow 4 \rightarrow 5 \\
\rightarrow 19 \rightarrow 18 & \rightarrow 17 \rightarrow 16 \rightarrow 21 \rightarrow 22 \rightarrow 17 \rightarrow 20 \rightarrow 25 \rightarrow 24 \\
\rightarrow & \rightarrow 12 \rightarrow 7 \rightarrow 2 \rightarrow 1\end{aligned}$ & $30 \%$ \\
\hline 7 & $\begin{aligned} & 1 \rightarrow 6 \rightarrow 7 \rightarrow 12 \rightarrow 17 \rightarrow 16 \rightarrow 21 \rightarrow 22 \rightarrow 17 \rightarrow 18 \\
\rightarrow & 19 \rightarrow 24 \rightarrow 25 \rightarrow 20 \rightarrow 15 \rightarrow 10 \rightarrow 5 \rightarrow 4 \rightarrow 3 \rightarrow 2 \rightarrow 1\end{aligned}$ & $15 \%$ \\
\hline 8 & $\begin{aligned} & 1 \rightarrow 2 \rightarrow 3 \rightarrow 8 \rightarrow 7 \rightarrow 12 \rightarrow 17 \rightarrow 18 \rightarrow 19 \rightarrow 24 \rightarrow 23 \rightarrow 22 \rightarrow 21 \\
\rightarrow & 16 \rightarrow 17 \rightarrow 23 \rightarrow 24 \rightarrow 25 \rightarrow 20 \rightarrow 15 \rightarrow 10 \rightarrow 5 \rightarrow 4 \rightarrow 3 \rightarrow 2 \rightarrow 1\end{aligned}$ & $10 \%$ \\
\hline & $\begin{aligned} 1 & \rightarrow 6 \rightarrow 11 \rightarrow 16 \rightarrow 21 \rightarrow 22 \rightarrow 17 \rightarrow 18 \rightarrow 19 \rightarrow 24 \\
\rightarrow 25 & \rightarrow 20 \rightarrow 15 \rightarrow 10 \rightarrow 9 \rightarrow 14 \rightarrow 13 \rightarrow 12 \rightarrow 7 \rightarrow 6 \rightarrow 1\end{aligned}$ & $5 \%$ \\
\hline 10 & $\begin{aligned} & \rightarrow \rightarrow 6 \rightarrow 11 \rightarrow 16 \rightarrow 17 \rightarrow 12 \rightarrow 7 \rightarrow 8 \rightarrow 9 \rightarrow 10 \rightarrow 5 \rightarrow 4 \rightarrow 9 \rightarrow 14 \\
& \rightarrow 19 \rightarrow 24 \rightarrow 25 \rightarrow 20 \rightarrow 24 \rightarrow 23 \rightarrow 18 \rightarrow 17 \rightarrow 22 \rightarrow 21 \rightarrow 16 \rightarrow 11 \rightarrow 6 \rightarrow 1\end{aligned}$ & $5 \%$ \\
\hline
\end{tabular}

stations to which the user was connected exists. Table II gives the sequence of connected base stations analogous to path number 1 in table I. Similarly, for each path, sequence of connected base stations can be derived.

TABLE II

SEQUENCE OF BASE STATIONS ANALOGOUS TO PATH

\begin{tabular}{|c|c|c|}
\hline Path Nr. & Sequence of base stations & \%age \\
\hline 1 & $\begin{array}{c}\mathrm{BS}_{1} \rightarrow B S_{2} \rightarrow B S_{9} \rightarrow B S_{10} \rightarrow B S_{11} \rightarrow B S_{12} \rightarrow B S_{8} \\
\text { ( } B S_{11} \rightarrow B S_{8} \rightarrow B S_{7} \rightarrow B S_{3} \rightarrow B S_{4} \rightarrow B S_{6} \rightarrow B S_{1}\end{array}$ & $10 \%$ \\
\hline
\end{tabular}

By monitoring a user for a specific duration, statistics on connected base stations and their sequence can be obtained. By utilizing such statistics, it is possible to derive the probability of transition into a next cell, based on simple Markov model [5] given by,

$$
P\left(C_{n} \rightarrow C_{n+1}\right)=\frac{N\left(C_{n} \rightarrow C_{n+1}\right)}{N\left(C_{n}\right)}
$$

Where, $C_{n} \rightarrow \mathrm{C}_{n+1}$ indicates transition from cell $_{n}$ to 
cell $_{n+1}, N($.$) indicates the number of times. Thus, by using$ mobility statistics, probability of transition into a next cell can be obtained as the ratio between; number of times user in cell $_{n}$ transited to cell $_{n+1}$ and number of times user was found in $\mathrm{cell}_{n}$.

Now, considering diurnal mobility of user, he traverses through specific landmarks on daily basis. These landmarks are associated with specific cells as shown in table III.

TABLE III

MAPPING LANDMARKS TO BASE STATIONS

\begin{tabular}{|c|c|c|c|c|c|c|c|}
\hline Landmark & Home & Office & University & Gym & NE & SW & SE \\
\hline Crossroad Nr. & 1 & 10 & 12 & 19 & 5 & 21 & 25 \\
\hline Base station Nr. & BS1 & BS10 & BS4 & BS8 & BS9 & BS3 3 & BS12 \\
\hline
\end{tabular}

By using the information about origin of a user (user originating from a specific landmark) the Markov based prediction can be extended as,

$$
P\left(C_{n} \rightarrow C_{n+1} / O\right)=\frac{N\left(C_{n} \rightarrow C_{n+1} / O\right)}{N\left(C_{n} / O\right)}
$$

Where $O$ indicates origin. Here, in cell $_{n}\left(C_{n}\right)$, only statistics of the user when originated from a specific landmark is considered instead of considering all transition history. For instance, consider figure 3 a), where user has arrived at cell 2 from office (cell 3). While predicting cell transition probabilities at cell 2, in simple markov case, all the user statistics are considered. Where as, in this case, only the statistical history of user in cell 2 when he had arrived from cell 3 are considered.

Similarly, by using information about both origin and destination of a user (user originating from a certain landmark and traveling to a specific landmark), the Markov based prediction can be further extended as,

$$
P\left(C_{n} \rightarrow C_{n+1} / O \& D\right)=\frac{N\left(C_{n} \rightarrow C_{n+1} / O \& D\right)}{N\left(C_{n} / O \& D\right)}
$$

Where $O$ indicates origin, $D$ indicates destination. Here, in cell $_{n}\left(C_{n}\right)$, only statistics of the user when originated from a specific landmark and going to a certain landmark is considered instead of considering all transition history. For instance, consider figure $3 \mathrm{~b}$ ), where user has arrived at cell 2 from office (cell 3) and will be further traveling to gym (cell 6). While predicting cell transition probabilities at cell 2, in simple Markov case, all the user statistics are considered. Where as, in this case, only the statistical history of user in cell 2 when he had arrived from cell 3 and traveled further to cell 6 are considered.

Obtaining information about destination becomes more feasible with advent of autonomous cars and technological advancements in vehicular positioning. With appropriate interface, such information can be imported by cellular networks and can be utilized to improve mobility prediction.

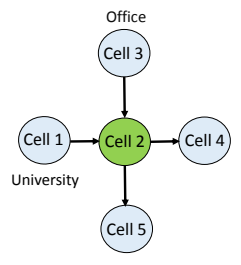

a) With origin

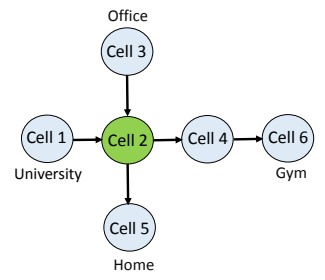

b) With origin and destination
Fig. 3. Markov chain with additional information

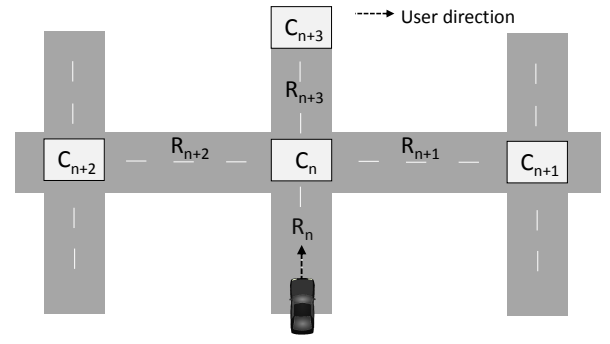

Fig. 4. Route map

\section{B. Route Prediction}

In this section, mobility prediction is more localized and instead of predicting the larger area (next cell), immediate future route is predicted. Figure 4 depicts the considerations of routes and crossroads in this work. Consider a user traveling in route $R_{n}$. At crossroad $C_{n}$ the user can take future routes $R_{n+1}$ or $R_{n+2}$ or $R_{n+3}$. Thus, route prediction in this work is to predict the next road user in $R_{n}$ would traverse, after forthcoming crossroad $C_{n}$.

A user could be monitored for several business days and statistics of different routes (sequence of crossroads) a user takes could be maintained. Such statistics enables deriving the probability of next road, based on simple Markov model [5] given by,

$$
P\left(R_{n} \rightarrow R_{n+1}\right)=\frac{N\left(R_{n} \rightarrow R_{n+1}\right)}{N\left(R_{n}\right)}
$$

Where, $R_{n} \rightarrow \mathrm{R}_{n+1}$ indicates transition from road $_{n}$ to $\operatorname{road}_{n+1}, N($.$) indicates the number of times. Thus, by using$ mobility statistics, probability of transition into a next road can be obtained as the ratio between; number of times user in road $_{n}$ transited to road $_{n+1}$ and number of times user was found in $\operatorname{road}_{n}$.

Now, assuming diurnal mobility of the user, specific landmarks are traversed by the user on daily basis. These landmarks are associated with specific crossroads as shown in table III. With the information about origin of a user (user originating from a specific landmark) the Markov based route prediction can be extended as,

$$
P\left(R_{n} \rightarrow R_{n+1} / O\right)=\frac{N\left(R_{n} \rightarrow R_{n+1} / O\right)}{N\left(R_{n} / O\right)}
$$


Where $O$ denotes a specific origin. In this case, while predicting next route before each crossroad $C_{n}$, only the statistics of user when originated from a specific landmark are considered. Contrastingly, in general Markov based prediction all of the user mobility statistics are considered, irrespective of where user originated.

Further, by knowing origin as well as the destination of a user, Markov based route prediction can be further extended as,

$$
P\left(R_{n} \rightarrow R_{n+1} / O \& D\right)=\frac{N\left(R_{n} \rightarrow R_{n+1} / O \& D\right)}{N\left(R_{n} / O \& D\right)}
$$

Where $O$ denotes a specific origin and $D$ denotes a specific destination.

Here, while predicting next route before each crossroad $C_{n}$, only the statistics of user when originated from a specific landmark and further traveled to a specific destination are considered. Contrastingly, in general Markov based prediction all of the user mobility statistics are considered, irrespective of which landmark user originated from and which landmark it further traveled to. In this case, it is assumed that user already knows origin and destination (one specific landmark out of all considered landmarks). Thus, user statistics which are recorded only when he originated from this origin and traveled to the specific destination are considered for deriving probabilities of next route.

\section{Context Exploitation}

In this section, route prediction is used in tandem with resource allocation to enable context awareness. In real world scenarios, there are several cases where the signal reception is very poor due to coverage holes (e.g., tunnels). The SINR of the user is very low in such coverage holes that the throughput of user drops to zero. Such cases are problematic for certain services such as streaming, full buffer services etc., in downlink. Consider figure 5, where a coverage hole (tunnel) is located in the road. The graphs (a) and (b) demonstrate dropping of SINR and throughput of user when traveling through the tunnel. Route prediction gives the probabilities $\left(P_{A}, P_{B}\right.$ and $\left.P_{C}\right)$ with which user might take next route. If route prediction indicates that road with the tunnel is the most probable next route, then context aware resource allocation is triggered. This procedure allocates more resources (physical resource blocks-PRBs) to the user and allows buffering of the data. This step is depicted in graph (c) of figure 5. Once the user is in tunnel, buffered data is used as depicted in graph (d) maintaining uniform service quality for streaming services. Figure 6 illustrates flowchart of the context aware resource allocation scheme. The actual user traversal in road with tunnel is confirmed to reduce unnecessary buffering of data due to false prediction (false alarm).

\section{Simulation Results}

The simulation set up follows system model described in section II. A 3D model following Madrid Grid layout is implemented in UNITY 3D as shown in figure 2. The base stations

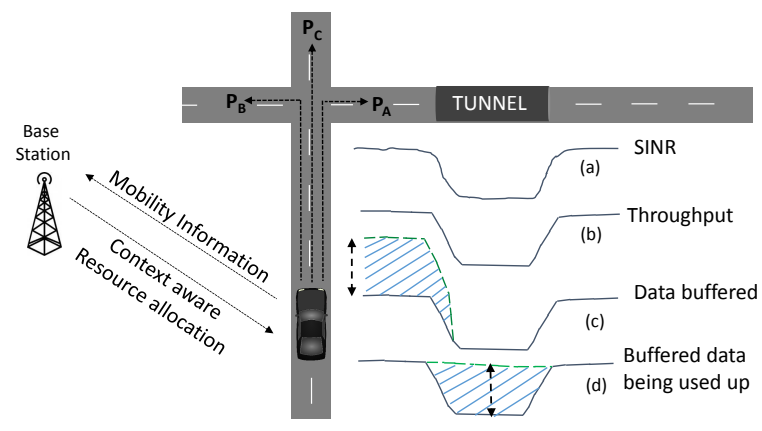

Fig. 5. Context aware resource allocation

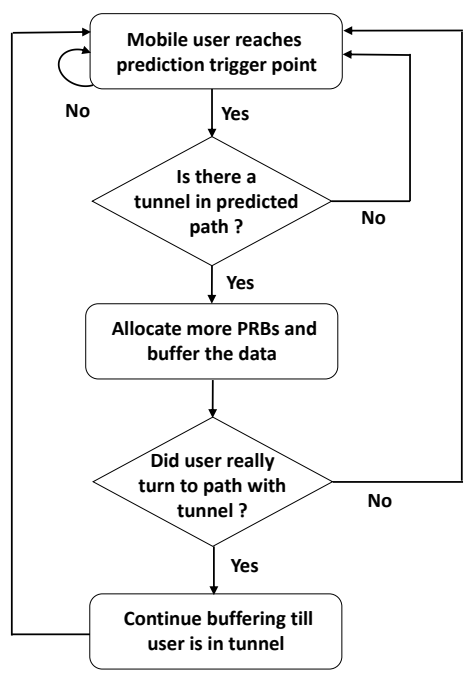

Fig. 6. Context aware resource allocation algorithm

are enabled with LTE technology and simulation parameters (E.g.,antenna parameters, pathloss models etc.) follow ITU-R, METIS guidelines [7]. The bandwidth is $10 \mathrm{MHz}$ (50 PRBs) and carrier frequency is $2 \mathrm{GHz}$. A car with average velocity of $30 \mathrm{~km} / \mathrm{h}$ is present and it can take trajectories as described in table II.

The statistics of user mobility and his connections to different base stations are obtained by running the simulations for 100 times. The user can traverse 10 set of trajectories with different probabilities as depicted in table I. Once the statistics are recorded, the above mentioned prediction schemes are carried out for 100 simulation runs and average accuracy of prediction is calculated. In learning phase (obtaining statistics) and in prediction phase, user traverses the same 10 paths with similar probabilities. Figure 7 a) compares accuracies of implemented next cell prediction schemes. The figure also includes two simple non-statistical methods (distance and geometry based) [8] of next cell prediction for comparison. The accuracy of next cell prediction was enhanced by using additional information of origin and destination, and was around 85\% surpassing rest of the schemes. Figure $7 \mathrm{~b}$ ) shows accuracies of route prediction methods. With additional information (origin, origin 
$\&$ destination), accuracy of prediction increases. The accuracy of prediction with information about origin \& destination is around $90 \%$.
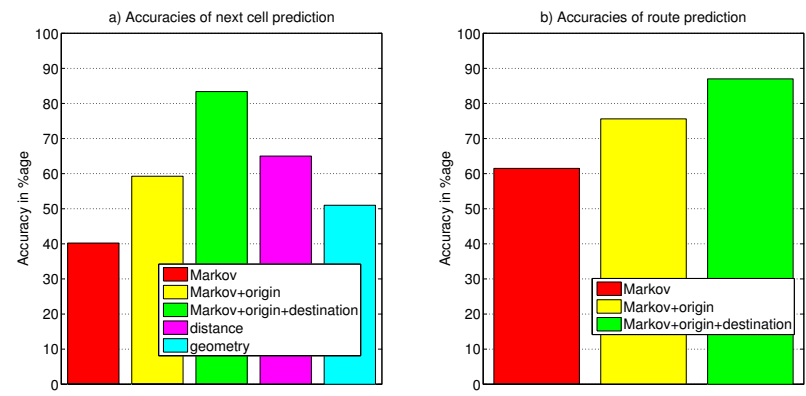

Fig. 7. Results of mobility prediction

Further, there are 6 coverage holes placed at different sites in simulation set up as depicted in figure 2. Context aware resource allocation is triggered by route prediction as discussed in sectionIII-C, by allocating more resource blocks (PRB $=2$ or PRB =3) and buffering data. The SINR to bit resource per element (BRE) mapping and throughput derivations follow [9]. The simulations are carried out for 100 runs and the average user throughput is obtained.
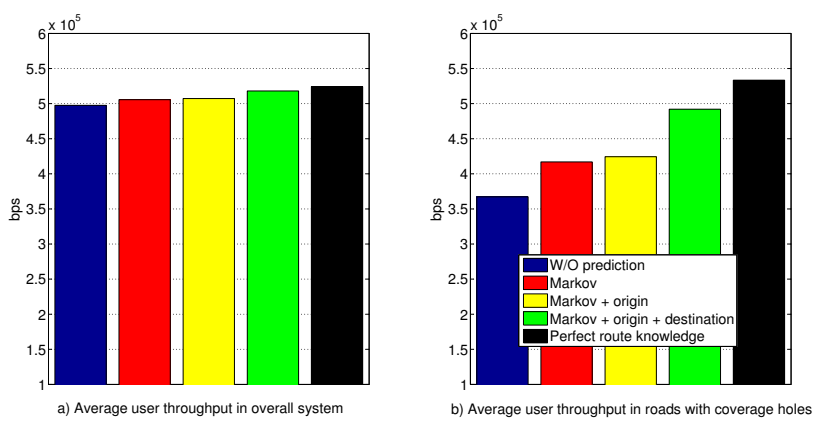

Fig. 8. Average throughput

Figure 8 a) shows the average user throughput in overall system. Figure $8 \mathrm{~b}$ ) shows average user throughput in roads with coverage hole. In both these cases $\mathrm{PRB}=2$, due to triggering of context aware resource allocation. Figure 9 a) compares percentages of user throughput improvement in roads with tunnel $(\mathrm{PRB}=2)$ and figure $9 \mathrm{~b}$ ) analyzes the same with $\mathrm{PRB}=3$. Average user throughput in roads with tunnel is improved when route prediction is deployed. The results get better with usage of additional information (origin, destination) for route prediction. In case of $\mathrm{PRB}=2$, around $35 \%$ improvement is seen and with $\mathrm{PRB}=3$, improvement of $65 \%$ is witnessed. The maximum possible improvements with perfect route knowledge is also depicted in these figures.

\section{CONCLUSION AND FUTURE WORK}

One of the key challenges for $5 \mathrm{G}$ mobile communications is to support high traffic volume while providing uniform service quality irrespective of high mobility. Thus, mobility prediction
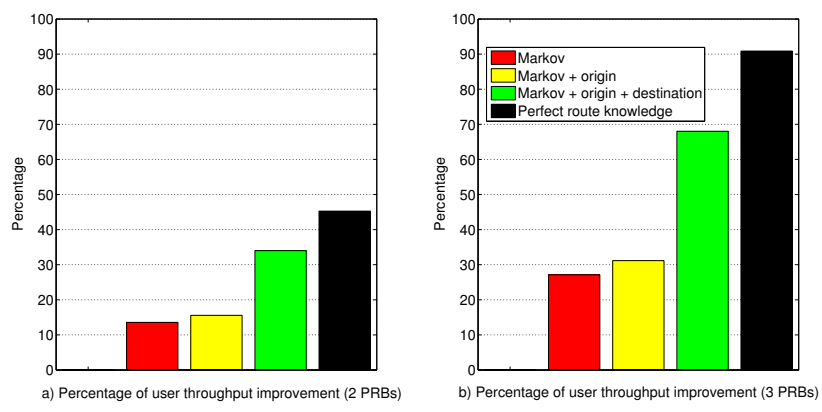

Fig. 9. Throughput improvement in paths with coverage hole (in percentage)

plays an important role in designing context aware procedures aiming to provide uniform service quality to users. Mobility of users is not random but direction oriented. Several users traverse similar trajectories on daily basis. This paper used information about such diurnal mobility of a user, his origin and destination, to enhance accuracy of mobility prediction in an urban scenario. Further, mobility prediction was used to proactively trigger context aware resource allocation. Thus, enabling uniform service quality for certain service classes in practical situations like coverage holes. Substantial improvements in average user throughput were observed in routes with coverage holes. Potential future work includes refining resource allocation scheme to target wide range of services, study of interfaces required to acquire information about user destination from vehicles, study of autonomous driving to obtain perfect route knowledge.

\section{ACKNOWLEDGMENT}

Part of this work has been performed in the framework of FP7 project ICT-317669 METIS II, which is partly funded by the European Union. The authors alone are responsible for the content of the paper.

\section{REFERENCES}

[1] Ericsson, More than 50 billion connected devices, white paper, Feb., 2011.

[2] METIS, D1.1 - Future radio access scenarios, requirements and KPIs, May, 2013, available online: https://www.metis2020.com.

[3] N.P. Kuruvatti; A. Klein; H.D. Schotten, Prediction of Dynamic Crowd Formation in Cellular Networks for Activating Small Cells, VTC-Spring, Glasgow,UK,2015

[4] A. Hadachi, O. Batrashev et al., Cell Phone Subscribers Mobility Prediction Using Enhanced Markov Chain Algorithm, Intelligent Vehicles Symposium, Michigan, USA, 2014

[5] S. Bellahsene and L. Kloul A New Markov-Based Mobility Prediction Algorithm for Mobile Networks, Conference on Computer Performance Engineering, Bertinoro, Italy, 2010.

[6] S. Michaelis, N. Piatkowski and K. Morik Predicting next network cell IDs for moving users with Discriminative and Generative Models, Mobile Data Challenge Workshop in conjunction with International Conference on Pervasive Computing, Newcastle, UK, 2012

[7] METIS, D6.1-Simulation Guidelines, May, 2013, available online: https: //www.metis2020.com.

[8] N.P. Kuruvatti, A. Klein, J. Schneider and H.D. Schotten, Exploiting Diurnal User Mobility for Predicting Cell Transitions, IEEE Globecom workshops, Atlanta, USA, 2013.

[9] M. Jar and G. Fettweis, Throughput Maximization for LTE Uplink via Resource Allocation, International Symposium on Wireless Communication Systems, Paris, France, 2012. 